\title{
Effects of Linear Feedback Trading in an Interactive Market Model
}

\author{
Michael Heinrich Baumann
}

\begin{abstract}
In the literature a lot of articles about so-called feedback-based trading strategies, i.e., strategies that compute investment exclusively from their own gain exist. Price is therein treated as a disturbance variable in the technical application. With these strategies, astonishing results may be shown. However, the so-called price taker property is always assumed, that means that one's own trading does not affect the price, which is quite unrealistic. In the work at hand, an interactive and thus more realistic market model is introduced which is completely determined by the traders' decisions. In this market model, distinct groups of traders can interact, e.g., feedback traders and noise traders, as they are considered to be relevant in economics in explaining real market prices. Furthermore, this interactive market model is a natural generalization of the geometric Brownian motion. Of course the model has to be bubble-prone to be realistic. The market model will be analyzed as well as the effects of linear feedback strategies and trading restrictions on the price. A way to calculate thresholds for the parameters to cause bubbles is provided. To conclude the work, backtesting on real market data is performed.
\end{abstract}

\section{MOTIVATION AND INTRODUCTION}

In recent years, a lot of papers about the use of feedbackbased trading strategies on financial markets, e.g. [4] and [5], were published. Therein, control theoretical ideas, well known in the engeneering sciences, have been adapted to financial market models. Trading strategies, i.e., the amount of bought and sold shares at each point of time, are defined without any assumptions about the market model, like the geometric Brownian motion or other stochastic models, and no enterprise data or business information is considered, thus, pure feedback strategies are applied. These methods are covered by the term "technical analysis". With this, remarkable results may be shown like, for example, the existence of arbitrage opportunities, see [3]. For obtaining these results, among a few technical assumptions also the so-called price taker property is assumed, which means, that one's own trading does not influence the price. This is, of course, a justified supposition if small investors are regarded, but usually these small investors do not use feedback strategies, as this would exceed their capabilities. However, controlbased trading is used by large investors like funds, which violate the price-taker assumption.

The work at hand provides a market model which does not only allow prices to be influenced by the traders but also that the price is determined by the traders' buying and selling decisions. The model will be discussed and different trading strategies will be established. All traders influence the price process and possibly, indirectly each other, too.

The author is with Faculty of Mathematics, Physics and Computer Science, University of Bayreuth, 95447 Bayreuth, Germany michael.baumann@uni-bayreuth.de
An important ingredient of our model are the so-called noise traders, who are non-sophisticated and act in a random way. By incorporating noise traders, uncertainty and risk is brought into the market. It will be shown that the resulting market model is a natural generalization of the widely used geometric Brownian motion. Furthermore, it is shown that feedback-based trading strategies are able to skew prices (which is in line with [7]) and even cause bubbles. It will be discused how technical trading restrictions must look like in order to prevent bubbles. The provided market model is not supposed to map real markets, but to analyze the effects of trading strategies.

\section{RELATED WORK}

\section{A. Feedback-based Trading Strategies}

It could be shown in the literature that the application of control technology to financial markets holds great potential, see [10] and [14]. Primbs und Barmish give an introduction to this topic in [19], which is further elaborated by Barmish in [2]. In [20], Primbs additionally picks up the idea of noise, but does not consider price trends caused by the traders. Further important literature about this subject is [8], [9], [11], [12], [18] and [22]. One basic technique discussed in the articles mentioned is the method of the so-called linear feedback controller, see Fig. 1,

$$
I_{t}^{F}:=I_{0}^{F}+K \cdot g_{t}^{F},
$$

where investment $I_{t}^{F}$ at time $t$ only depends on gain or loss $g_{t}^{F}$, initial investment $I_{0}^{F}$ and the feedback parameter $K>0$. Control theory is able to give mathematically rigorous propositions about the application of control-based trading strategies, e.g., concerning arbitrage possibilities. Therein, the price process $p_{t}$ of the underlying stock does not play a part directly and is rather treated like a disturbance variable in the technical implementation. Particularly, the price is not supposed to evolve according to a particular model. Using the means of control theory, astonishing conclusions are possible: For continuously differentiable prices a controller may be constructed which almost surely achieves positive return ([1], [3] and [4]) and still has a positive expectation

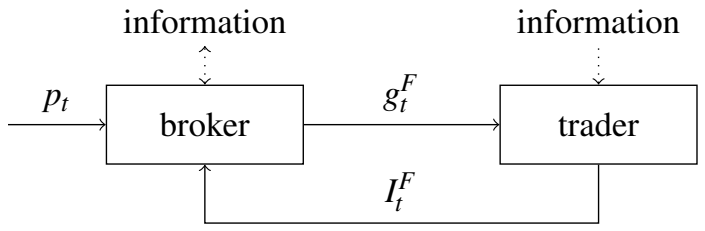

Fig. 1. Schematical interaction between broker and feedback trader 
for the gain under the assumption that the price evolves according to a geometric Brownian motion ([3]). Even when applied on real historic price data, such feedback strategies yield positive profits on the average, not mentioned yet that there exist a lot of optimization possibilities for them. In [16], the topic of positive profits is treated analytically, whereas in [5] and [15] it is dealt with via simulation.

Among other technical assumptions, the so-called price taker property, which implies that one's own trading does not affect the price, is presupposed. As the authors of the respective papers often point out, this is a very restrictive assumption as control-based trading strategies are mostly performed by large funds. These funds are able to influence the price in such a way, that during a transaction the price changes because of their actions, e.g., while selling of a lot of shares the ones selled first gain a higher price than whose selled last (see [15]).

\section{B. Noise}

In the field of game theory, market prices are determined by the traders' actions, but considering solely rational traders observed prices can only be explained insufficiently (see [21]). E.g., a fund investing previously defined amounts in certain stocks should always be worth as much as the corresponding stocks. But usually this is not observed in real markets, as funds are typically underestimated. One possibility to explain this is to assume so-called noise, which means unpredictable distortions (see [13] and [17]). Noise can be brought into the model either by defining prices with random fluctuations or by letting the traders make decisions that are irrational from an objective point of view. This, again, may be caused by noisy information which the traders take for real. If equilibrium prices are computed on markets including these so-called noise traders, a lot of phenomena observable on real markets can be explained, as Black describes in [6].

\section{MARKET MODEL}

\section{A. Pricing}

We are going to derive a market model, i.e., a price model or price process $p_{t}$, respectively, on a discrete time grid $\mathscr{T}=$ $\{0,1, \ldots, T\}$ or $\mathscr{T}=\mathbb{N}_{0}$, in which price is determined by buying and selling decisions $\Delta I_{t}$ of the traders. In order to keep the exposition simple, in this paper wie restrict our analysis to two types of traders, feedback traders $(F)$ and noise traders $(N)$. Thus, $\Delta I_{t}$ can consist of buying and selling decisions of the two groups of traders, that is

$$
\Delta I_{t}:=\Delta I_{t}^{F}+\Delta I_{t}^{N} .
$$

The total investment of the trading groups at time $t$ is given through $I_{t}=\sum_{i=0}^{t} \Delta I_{i}, I_{t}^{F}=\sum_{i=0}^{t} \Delta I_{i}^{F}$ and $I_{t}^{N}=\sum_{i=0}^{t} \Delta I_{i}^{N}$. The market model should satisfy the following claims: If supply equals demand, the prices remain stable:

$$
\Delta I_{t}=0 \Rightarrow p_{t+1}=p_{t}
$$

If supply exceeds demand, prices decline and vice versa:

$$
\Delta I_{t} \rightarrow \infty \Rightarrow p_{t+1} \rightarrow \infty, \Delta I_{t} \rightarrow-\infty \Rightarrow p_{t+1} \rightarrow 0 \text { and }
$$

$p_{t+1}$ strictly monotonic increasing in $\Delta I_{t}$

The exponential function defined by

$$
p_{t+1}:=p_{t} \cdot e^{M^{-1} \cdot \Delta I_{t}}=p_{0} \cdot e^{M^{-1} \cdot I_{t}}
$$

meets these requirements where $M>0$ is some scaling factor for trade volume and $p_{0}>0$ indicates the initial price. The gain $g_{t}^{\ell}$ of a trader at time $t$ is calculated as

$$
g_{t}^{\ell}:=g_{t-1}^{\ell}+I_{t-1}^{\ell} \cdot \frac{p_{t}-p_{t-1}}{p_{t-1}}=\sum_{i=1}^{t} I_{i-1}^{\ell} \cdot \frac{p_{i}-p_{i-1}}{p_{i-1}},
$$

where $I_{t}^{\ell}$ is this trader's specific investment. If only one trader operated on the market it can be recognized that $\Delta I_{t}^{\ell} \rightarrow \pm \infty$ leads to $g_{t+1}^{\ell} \rightarrow+\infty$, as it is

$$
g_{t+1}^{\ell}=g_{t}^{\ell}+\left(I_{t-1}^{\ell}+\Delta I_{t}^{\ell}\right)\left(e^{M^{-1} \cdot \Delta t_{t}^{\ell}}-1\right) .
$$

This implies that, this market model is bubble-prone. In the following, three aspects of the model will be discussed. Uncertainty has to be added to the model, infinite investments are unrealistic and trading restrictions have to be established.

\section{B. Noise-Trading and Relation to Geometric Brownian Mo-} tion

Now, trading strategies for the so-called noise traders have to be determined, that are supposed to be random but should follow a certain distribution, i.e., they should not be idiosyncratic. We set

$$
\Delta I_{t}^{N}:=M \cdot\left(\left(\mu-\frac{\sigma^{2}}{2}\right)+\sigma \cdot \Delta W_{t}\right),
$$

where $M>0$ is the scaling parameter for volumes of trade ${ }^{1}$ from (3), $\mu \in \mathbb{R}$ can be interpreted as saving deposit per time step, $\sigma>0$ specifies the so-called volatility of the market, $\Delta W_{t} \stackrel{\text { iid }}{\sim} \mathscr{N}(0,1)$ is a random walk that brings noise into the market and $-\frac{\sigma^{2}}{2}$ can be interpreted as risk aversion. This choice of (5) leads to the result that the market model (3) is a natural generalization of the geometric Brownian motion $(\mathrm{GBM})^{2}$, as the following lemma shows if we assume that there are no feedback traders acting on the market.

Lemma 1: For $I_{t}^{F} \equiv 0$ and for all $M>0$ the paths $p_{t}$ of the price process follow the paths of the geometric Brownian motion.

Proof: For this proof (2),(3) and (5) are combined. First of all, it is computed

$$
\begin{aligned}
I_{t}^{N} & =\sum_{i=0}^{t} \Delta I_{t}^{N}=M \cdot \sum_{i=0}^{t}\left(\left(\mu-\frac{\sigma^{2}}{2}\right)+\sigma \cdot \Delta W_{t}\right) \\
& =M \cdot\left(\left(\mu-\frac{\sigma^{2}}{2}\right)(t+1)+\sigma \cdot W_{t+1}\right),
\end{aligned}
$$

where $W_{t}$ is a Wiener process. The price at time $t$ is

$$
p_{t}=p_{0} \cdot e^{M^{-1} \cdot\left(I_{t-1}^{N}+I_{t-1}^{F}\right)}=p_{0} \cdot e^{\left(\mu-\frac{\sigma^{2}}{2}\right) t+\sigma \cdot W_{t}},
$$

${ }^{1} M \cdot \sum_{\mathscr{T}}\left|\Delta I_{t}^{N}\right|=\sum_{\mathscr{T}}$ "trade volume" ${ }_{t}$

${ }^{2}$ The GBM is the solution of the stochastic differential equation $\mathrm{d} S_{t}=$ $\mu S_{t} \mathrm{~d} t+\sigma S_{t} \mathrm{~d} W_{t}$. One can show that $S_{t}=S_{0} \cdot e^{\left(\mu-\frac{\sigma^{2}}{2}\right) t+\sigma \cdot W_{t}}$. 
which is also the formula for the geometric Brownian motion.

\section{EFFECTS OF FEEDBACK-BASED TRADING}

In this section it shall be analyzed how our market model behaves if there is a linear feedback trader (1) acting on the market. By considering formula (4) for gain and loss of the respective traders, a result about the relation between price and investment of linear feedback traders follows.

Lemma 2: The relative change in the investment of linear feedback traders for all price processes $p_{t}$ is a linear function of the relative changes of the price process if $\forall t I_{t}^{F}>0$, i.e.,

Proof: It is

$$
\frac{I_{t}^{F}-I_{t-1}^{F}}{I_{t-1}^{F}}=K \cdot \frac{p_{t}-p_{t-1}}{p_{t-1}} .
$$

$$
\begin{aligned}
I_{t}^{F}-I_{t-1}^{F} & =K \cdot\left(g_{t}^{F}-g_{t-1}^{F}\right) \\
& =K \cdot I_{t-1}^{F} \cdot \frac{p_{t}-p_{t-1}}{p_{t-1}}
\end{aligned}
$$

Dividing by $I_{t-1}^{F}$ leads to the assertion.

\section{A. Purely Linear Feedback Controller}

In this subsection markets with purely linear feedback traders shall be studied, that means $I_{t}^{N} \equiv 0$. In this case, the feedback-based investment strategy is given by

$$
\begin{aligned}
& I_{0}^{F}>0 \\
& I_{1}^{F}=I_{0}^{F}+K \cdot I_{0}^{F} \cdot\left(e^{M^{-1} I_{0}^{F}}-1\right) \text { and } \\
& I_{t}^{F}=I_{t-1}^{F}+K \cdot I_{t-1}^{F} \cdot\left(e^{M^{-1} \cdot\left(I_{t-1}^{F}-I_{t-2}^{F}\right)}-1\right), t \geq 2 .
\end{aligned}
$$

The assumption $K>0$ can be changed to $K \in(0,1]$ without any changes in the results. This would be more realistic because the traders do not have to find any credit bank if they have $I_{0}^{F}$ at their disposal. That means, every trader is just reinvesting a part of his gain. In all cases the properties of this market can be shown.

Lemma 3: If $I_{t}^{N} \equiv 0$, the investment $I_{t}^{F}$ of the linear feedback trader is strictly increasing in $t\left(\Delta I_{t}^{F}>0\right)$.

Proof: The lemma is proven by induction. Because of $e^{M^{-1} I_{0}^{F}}>1$ and $I_{0}^{F}>0$, the initial inequalitiy $I_{1}^{F}>I_{0}^{F}$ is true. The induction step follows, as $e^{M^{-1}\left(I_{t-1}^{F}-I_{t-2}^{F}\right)}>1$ and $I_{t-1}^{F}>I_{t-2}^{F}>\ldots>0$.

It follows that $I_{t}^{F}>0$ because of $I_{0}^{F}>0$. This means that feedback traders' investment will increase prices and thus also their gain. This leads to more investment and so on. But this does not necessarily have to end in a bubble ${ }^{3}$, because the investment can converge to some value like in Fig. 2 on the left side. It is possible to calculate thresholds for the initial investment so that feedback trading will cause a bubble like in Fig. 2 on the right side 4 .

Theorem 1: If $I_{t}^{N} \equiv 0$ and $\exists t^{*} \in \mathscr{T}: \Delta I_{t^{*}+1}^{F}>\Delta I_{t^{*}}^{F}$ then

$$
\Delta I_{t+1}^{F}>\Delta I_{t}^{F}
$$

\footnotetext{
${ }^{3}$ We say that a bubble occurs if $\exists t^{*}: \Delta I_{t+1}>\Delta I_{t} \forall t \geq t^{*}$.

${ }^{4}$ Clearly, because the model is in discrete time, for all $t \in \mathscr{T}$ the price is $<\infty$.
}
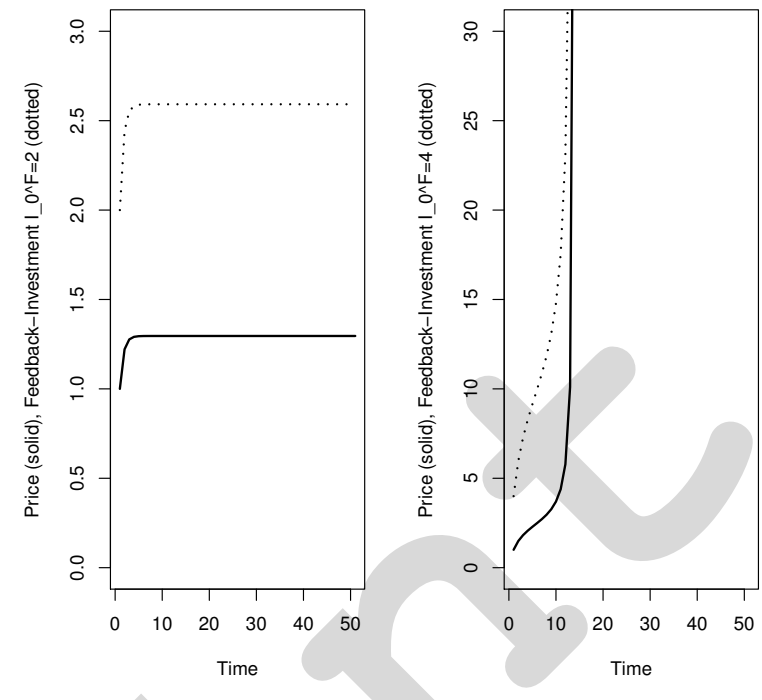

Fig. 2. Only linear feedback trader causing no bubble (left) and causing a bubble (right) (parameters: $T=250, p_{0}=1, M=5, K=1, I_{0}^{F}=2$ (left), $I_{0}^{F}=4$ (right); outputs: $g_{T}^{F}=0.592$ (left), $g_{T}^{F}=\mathrm{NaN}$ (right))

holds for all $t \geq t^{*}$. That means, the buyed amount of stocks $\Delta I_{t}^{F}$ of the feedback trader is strictly increasing for all $t \geq t^{*}$.

Proof: The induction step

$$
\Delta I_{t}^{F}>\Delta I_{t-1}^{F} \Rightarrow \Delta I_{t+1}^{F}>\Delta I_{t}^{F}, t \geq 1,
$$

has to be shown. This is true because of

$$
\Delta I_{t+1}^{F}>\Delta I_{t}^{F} \Leftrightarrow I_{t}^{F} \cdot\left(e^{M^{-1} \cdot \Delta I_{t}^{F}}-1\right)>I_{t-1}^{F} \cdot\left(e^{\left.M^{-1} \cdot \Delta I_{t-1}^{F}-1\right),}\right.
$$

Lemma 3 and the induction hypothesis.

To calculate a threshold for $I_{0}^{F}$ different cases are possible: One is, that the bubble starts at $t=0$, that means:

$$
\begin{array}{ll} 
& \Delta I_{1}^{F}>\Delta I_{0}^{F} \\
\Leftrightarrow & I_{1}^{F}-2 \cdot I_{0}^{F}>0 \\
\Leftrightarrow & K \cdot I_{0}^{F} \cdot\left(e^{M^{-1} \cdot I_{0}^{F}}-1\right)-I_{0}^{F}>0 \\
\Leftrightarrow & I_{0}^{F}>M \cdot \ln \left(1+\frac{1}{K}\right)
\end{array}
$$

For $K=1$ and $M=10$ this leads to $I_{0}^{F}>6.932$. But a bubble can also arise, when

$$
\begin{aligned}
& \Delta I_{2}^{F}>\Delta I_{1}^{F} \\
\Leftrightarrow & K \cdot I_{1}^{F} \cdot\left(e^{M^{-1} \cdot\left(I_{1}^{F}-I_{0}^{F}\right)}-1\right) \\
\Leftrightarrow & K \cdot\left(I_{0}^{F}+K \cdot I_{0}^{F} \cdot\left(e^{M^{-1} \cdot I_{0}^{F}}-1\right)\right)\left(e^{M^{-1} \cdot K \cdot I_{0}^{F}\left(e^{M^{-1}} \cdot I_{0}^{F}-1\right)}-1\right) .
\end{aligned}
$$

One calculates for $K=1$ and $M=10$ that this holds if $I_{0}^{F}>$ 5.071. Hence, the threshold is:

$$
I_{0}^{F * *}=\inf \left\{I_{0}^{F *} \mid \exists t: \Delta I_{t+1}^{F}>\Delta I_{t}^{F} \forall I_{0}^{F}>I_{0}^{F *}\right\}
$$

By simulation it turns out that $I_{0}^{F * *} \approx 3.680$ for $K=1$ and $M=10$. Thus, no infinite investments are necessary to generate infinite gain. Even with a relatively moderate initial 
budget, financial bubbles are able to occur. At any rate, if a bubble occurs, it cannot be stopped. It is still quite unrealistic that the market development is deteministic, which is caused by the fact, that only one trader is acting on the market. In the following subsection, noise traders and feedback traders act simultaneously on the same market.

\section{B. Linear Feedback Controller in a Noisy Market}

Now, the behaviour of linear feedback traders in noisy markets shall be investigated. It is

$$
p_{t}=p_{t-1} \cdot e^{M^{-1} \cdot\left(\Delta I_{t-1}^{N}+\Delta I_{t-1}^{F}\right)} .
$$

This may be rewritten to

$$
p_{t}=p_{t-1} e^{\left(\mu-\frac{\sigma^{2}}{2}\right)+\sigma \cdot \Delta W_{t-1}} e^{M^{-1} \cdot \Delta I_{t-1}^{F}}
$$

with

$$
e^{\left(\mu-\frac{\sigma^{2}}{2}\right)+\sigma \cdot \Delta W_{t-1}} \stackrel{\text { iid }}{\sim} \mathscr{L} \mathscr{N}\left(\mu-\frac{\sigma^{2}}{2}, \sigma^{2}\right)
$$

and $\mathscr{L} \mathscr{N}$ describing the lognormal distribution. One conclusion is the following:

$$
\mathbb{E}\left[e^{\left(\mu-\frac{\sigma^{2}}{2}\right)+\sigma \cdot \Delta W_{t-1}}\right]=e^{\mu-\frac{\sigma^{2}}{2}+\frac{\sigma^{2}}{2}}=e^{\mu}
$$

The dynamic of $I_{0}^{F}$ can be written as

$$
\begin{aligned}
& I_{0}^{F}>0 \\
& \begin{aligned}
I_{1}^{F}= & I_{0}^{F}+K \cdot I_{0}^{F} \cdot\left(e^{\left(\mu-\frac{\sigma^{2}}{2}\right)+\sigma \cdot \Delta W_{t-1}} \cdot e^{M^{-1} \cdot I_{0}^{F}}-1\right) \text { and } \\
I_{t}^{F}=I_{t-1}^{F}+K \cdot I_{t-1}^{F} & \quad\left(e^{\left(\mu-\frac{\sigma^{2}}{2}\right)+\sigma \cdot \Delta W_{t-1}} \cdot e^{M^{-1} \cdot\left(I_{t-1}^{F}-I_{t-2}^{F}\right)}-1\right), t \geq 2 .
\end{aligned}
\end{aligned}
$$

Analogous to the market model without noise it follows:

Lemma 4: If $\mu \geq 0$, the investement of linear feedback traders $I_{t}^{F}$ is strictly monotonously increasing in expectation, i.e.,

$$
\mathbb{E}\left[I_{t+1}^{F}\right]>I_{t}^{F}
$$

at time $t$.

Proof: The statement of the lemma is a consequence of the proof of Lemma 3 and (6).

Furthermore, the following can be shown.

Theorem 2: If $\Delta I_{t}^{F}>\Delta I_{t-1}^{F}$ and $\Delta I_{t}^{F}>0$ it holds that

$$
\mathbb{E}\left[\Delta I_{t+1}^{F}\right]>\Delta I_{t}^{F}
$$

at time $t$ provided $\mu \geq 0$.

Proof: The statement is proved like Theorem 1 under application of (6).

In this market model, no fixed thresholds may be specified but only expected ones. Simulations show that bubbles do not need to occur (see Fig. 3) but have the chance to do so (see Fig. 4). Also, bubbles may burst in the case that accidentally noise traders disinvest more than feedback controllers invest. However, this becomes more unlikely (because of the small tails of the normal distribution) the larger the bubbles are. That is why the next section will show how to prevent bubbles. Clearly, in real markets some economic processes, e.g., market regulations, would start if bubbles occur and could let them burst which are not in the scope of this paper.

\section{Trading Restrictions}

To avoid bubbles, $\Delta I_{t}^{F}$ will be limited so that

$$
-b \leq \Delta I_{t}^{F} \leq b
$$

for $b>0$. It is supposed that one linear feedback trader and a lot of noise traders act on the market and the investment of the noise traders sums up to $I_{t}^{N}$. Thus, $\left|\Delta I_{t}^{N}\right|>b$ is possible. Further, (1) and (4) are generalized for $I_{0}^{F}>0$ to

$$
I_{t}^{b F}=\max \left\{\min \left\{I_{0}^{F}+K g_{t}^{b F}, I_{t-1}^{b F}+b\right\}, I_{t-1}^{b F}-b\right\},
$$

where

$$
g_{t}^{b F}=g_{t-1}^{b F}+I_{t-1}^{b F} \cdot \frac{p_{t}-p_{t-1}}{p_{t-1}} .
$$

This implies $I_{0}^{b F}=\max \left\{I_{0}^{F}, b\right\}$. Through simulation, for this controller Fig. 5 was created, where it can be recognized that bubbles can occur and burst afterwards again. This is possible because for the limited $\Delta I_{t}^{F}$ the noise traders have the chance to counteract the investment of the feedback traders, i.e., that despite of large gains of the feedback traders the price has a chance to fall. This may be written as:

$$
\mathbb{P}\left(\left(\mu-\frac{\sigma^{2}}{2}\right)+\sigma \cdot \Delta W_{t}+M^{-1} \cdot b<0\right) \geq \alpha \in(0,1)
$$

which is equivalent to

$$
\Phi\left(\frac{\left(\mu-\frac{\sigma^{2}}{2}\right)+M^{-1} \cdot b}{\sigma}\right) \leq 1-\alpha
$$

and

$$
b \leq M \cdot\left(\sigma \cdot \Phi^{-1} \cdot(1-\alpha)-\left(\mu-\frac{\sigma^{2}}{2}\right)\right),
$$

where $\Phi$ denotes the distribution function of the standardnormal distribution. However, such $b$ does not have to exist. The next section is about to discover the effects of several trading restrictions on bubble occurring using real market data.

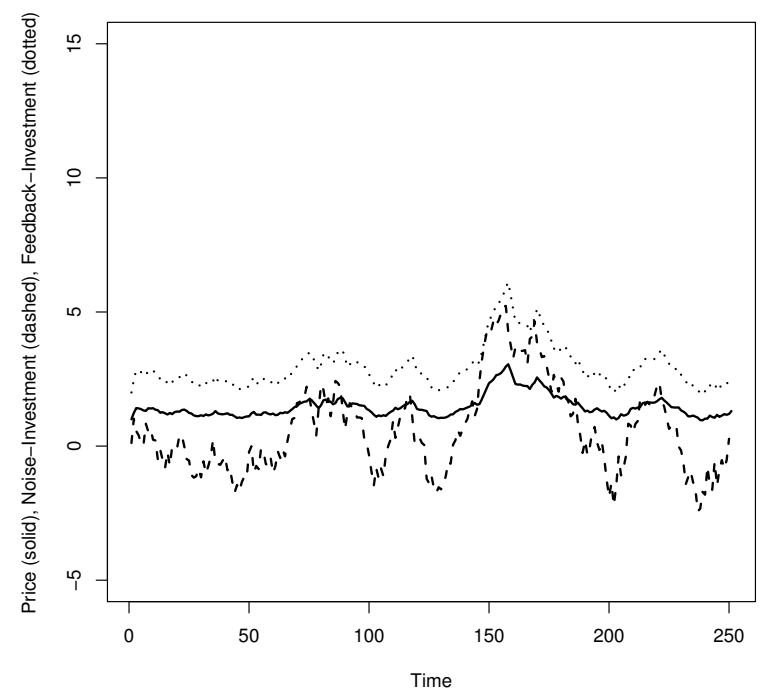

Fig. 3. Linear feedback trader in a noisy market not causing a bubble (parameters: $T=250, p_{0}=1, M=5, \mu=0.03 / 360, \sigma=0.05, K=1$, $I_{0}^{F}=2$; output: $g_{T}^{F}=0.396$ ) 


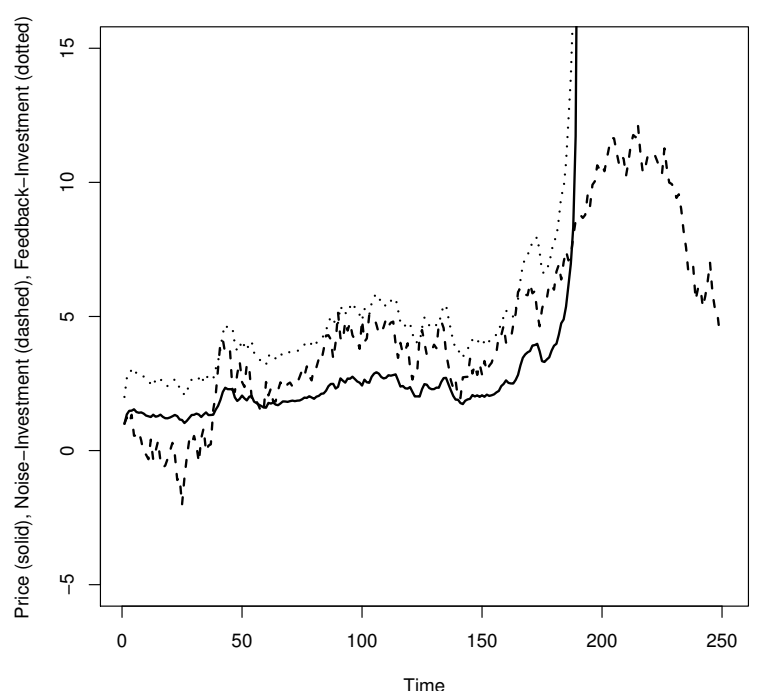

Fig. 4. Linear feedback trader in a noisy market causing a bubble (parameters: $T=250, p_{0}=1, M=5, \mu=0.03 / 360, \sigma=0.05, K=1$, $I_{0}^{F}=2$; output: $\left.g_{T}^{F}=\mathrm{NaN}\right)$

\section{BACKTESTING}

The purpose of this section is to examine the opening XET-price of the "Deutsche Bank AG" stock between 201308-01 and 2014-07-31, taken from Thomson Reuters Datastream 5.1, and how it would have developed according to the presented model if several feedback traders influenced the market. In doing so, various initial investments ${ }^{5}$ and trading restrictions are studied. For this, $\Delta I_{t}^{N}$ is computed from the stock data, which is no longer random, but fixed and, however, unknown to the feedback trader. In Fig. 6 the impact of different initial investments $\left(1 \cdot 10^{9}, 0.2 \cdot 10^{10}\right.$ and $0.5 \cdot 10^{10}$ ) together with the uniform trading restriction $b=1 \cdot 10^{10}$ is shown. As it was expected, the price gets more distorted with higher investment. In this test series, only for an enormous investment of $0.5 \cdot 10^{10}$ a bubble occurs ${ }^{6}$.

In Fig. 7 the effect of trading restrictions on the market is examined. To this end, the initial investment is fixed $\left(I_{0}^{F}=\right.$ $\left.0.5 \cdot 10^{10}\right)$ and the bound $b$ varies $\left(1 \cdot 10^{8}, 1 \cdot 10^{9}\right.$ and $\left.1 \cdot 10^{10}\right)$. One result is that through trading restrictions the occurrence of bubbles can be prevented and that bubbles can be let burst. In this example, the scaling factor for the trade volume was set to $M=13403793634$. By combining (2), (3) and (5), it can be shown that a feedback trader needs the more initial investment to push prices the higher the trade volume of the specific stock is.

\section{CONCLUSIONS AND FUTURE WORKS}

\section{A. The nature of bubbles}

In the work at hand, we have seen that the indroduction of linear feedback traders in a simple market model may

\footnotetext{
${ }^{5}$ The currency unit in this section is the Euro (EUR, €).

${ }^{6}$ For comparison: The market capitalisation of the "Deutsche Bank AG" on 2013-08-01 was circa $0.472 \cdot 10^{11}$.
}

cause bubbles to appear. At a first glance, this seems to be some kind of "money press". But, even if a trader has nearly infinite gain, this money is invested in stocks. For actually getting cash, a buyer is needed and to find such one could be a hard task. Because, if the supposed buyer notices that he is going to trade on a financial bubble he will not invest. Further on, selling the stocks to make money will let the bubble burst.

\section{B. Conclusions}

All in all, it can be seen that the basic dynamic of the market, i.e., that rising demand leads to rising prices, together with the linear feedback controller may cause bubbles as increasing prices with high investments lead to higher profits and thus again to more investments and increasing prices. To produce bubbles, however, no high investments - in relative terms - are necessary. This changes when uncertainty and risk enter the market. To influence the market significantly in this noisy setting enough market power is needed, that means that one's own investment in relation to that of the noise traders' may not be too low. That means, the higher the trading volume the greater the needed initial investment. One idea to avoid bubbles or to let them burst is to impose trading restrictions. In summary, the presented market model is suitable to analyze effects of investments and disinvestments and the occurrence of bubbles, because the so-called pricetaker property of the traders is removed.

\section{Future Works}

Future works may concentrate on the analysis of other (feedback) strategies in the market model provided in the work at hand. For example, including fundamentalists or analyzing the simultaneous long short controller would be

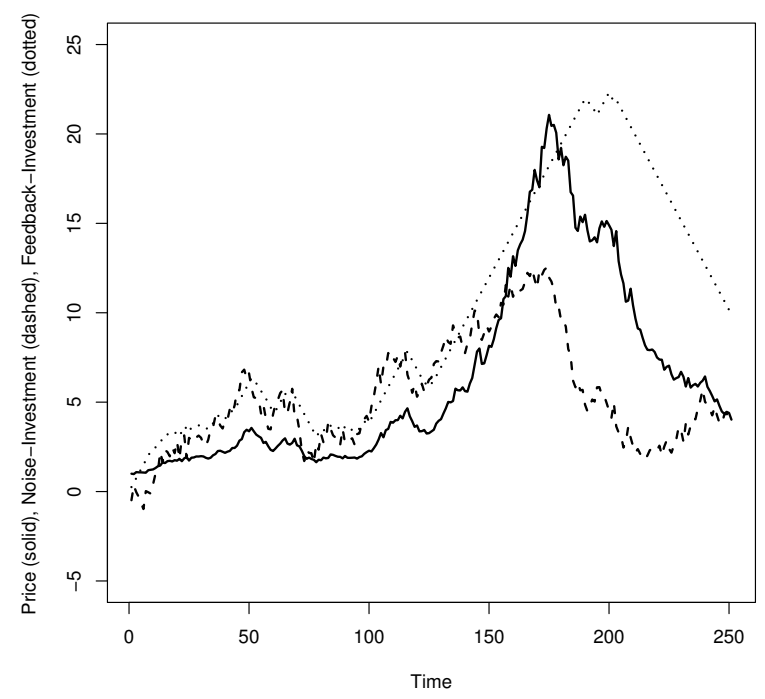

Fig. 5. Bounded linear feedback trader causing a bursting bubble (parameters: $T=250, p_{0}=1, M=5, \mu=0.03 / 360, \sigma=0.05, K=1, I_{0}^{F}=2$, $b=0.25$; outputs: $g_{T}^{F}=0.187, \max p_{t}=21.068, \max g_{t}^{F}=26.787$ ) 
of interest. This sort of controller invests in long and short investments at the begining and subsequently shifts the investments to that side with the greater gains. Another field of interest could be the construction of more game-theoretic market models in which (noisy) equilibrium strategies and their corresponding prices can be calculated. In this setting, the interaction of feedback based trading and equilibrium strategies of sophisticated traders or noisy strategies, which are on average equilibrium strategies, can be studied.

\section{ACKNOWLEDGMENTS}

The author wishs to thank Hanns-Seidel-Stiftung e.V. (HSS). Furthermore, the author would like to extend his thanks to Michaela Baumann, Bernhard Herz and Lars Grüne, all with University of Bayreuth.

\section{REFERENCES}

[1] B.R. Barmish, "On Performance Limits of Feedback Control-Based Stock Trading Strategies", Proceedings of the American Control Conference, 2011.

[2] B.R. Barmish, "On trading of equities: A robust control paradigm", Proceedings of the 17th World Congress, The International Federation of Automatic Control, 2008.

[3] B.R. Barmish and J.A. Primbs, "On Arbitrage Possibilities Via Linear Feedback in an Idealized Brownian Motion Stock Market", Proceedings of the IEEE Conference on Decision and Control, 2011.

[4] B.R. Barmish and J.A. Primbs, "On Market-Neutral Stock Trading Arbitrage Via Linear Feedback", American Control Conference, 2012.

[5] B.R. Barmish and J.A. Primbs and S. Malekpour and S. Warnick, "On the Basics for Simulation of Feedback-Based Stock Trading Strategies: An Invited Tutorial Session", 52nd IEEE Conference on Decision and Control, 2013.

[6] F. Black, "Noise", The Journal of Fincance, vol. XLI, issue 3, 1986.

[7] C. Bauer and P. De Grauwe and S. Reitz, "Exchange rate dynamics in a target zone - a heterogenous expectations approach", Deutsche Bundesbank Eurosystem, Discussion Paper, Series 1: Economic Studies, No $11 / 2007$.

[8] G.C. Calafiore, "An Affice Control Method for Optimal Dynamic Asset Allocation with Transaction Costs", Society for Industrial and Applied Mathematics, J. Control Optim., vol. 48, issue 4, 2009, pp 2254-2274.

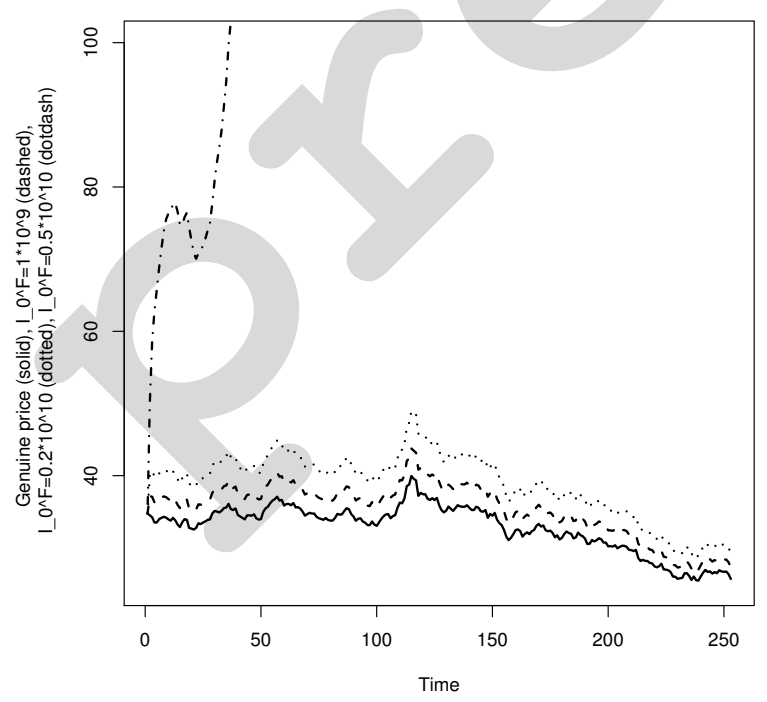

Fig. 6. Price $[€]$ of the "Deutsche Bank AG", partly distorted by linear feedback traders (parameters: $T=252, p_{0}=34.765, M=13403793634$, $K=1, b=1 \cdot 10^{10}$ )

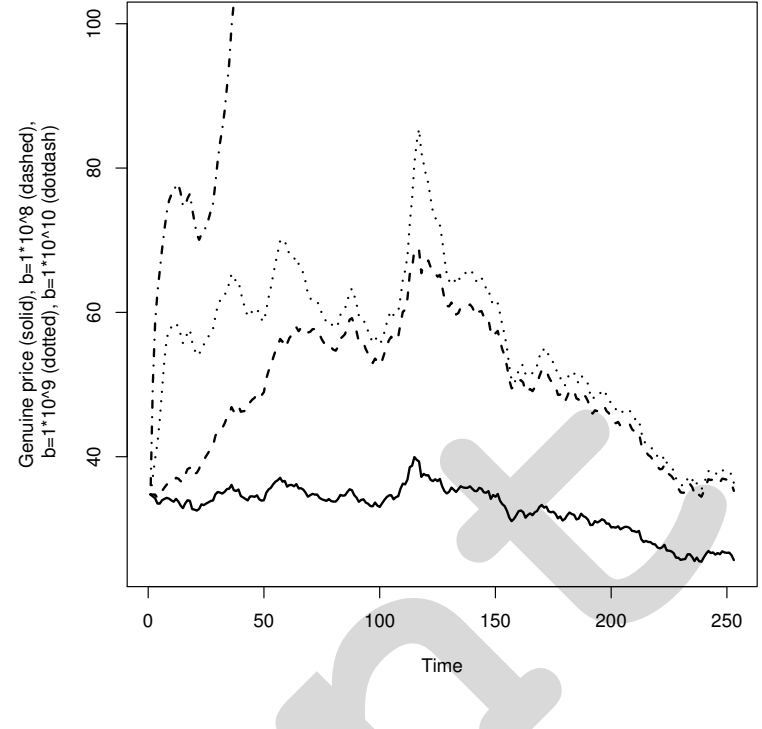

Fig. 7. Effects of trading restrictions on the distortion of the price $[€]$ of the "Deutsche Bank AG" caused by linear feedback traders (parameters: $\left.T=252, p_{0}=34.765, M=13403793634, K=1, I_{0}^{F}=0.5 \cdot 10^{10}\right)$

[9] G.C. Calafiore, "Mulit-period portfolio optimization with linear control policies", Automatica, vol. 44, 2008, pp 2463-2473.

[10] G.C. Calafiore and B. Monastero, "Data-driven asset allocation with guaranteed short-fall probability", American Control Conference, 2012.

[11] T.M. Cover, "Universal Portfolios", Mathematical Finance, vol. 1, issue 1, 1991, pp 1-29.

[12] T.M. Cover and E. Ordentlich, "Universal Portfolios with Side Information", IEEE Transactions on Information Theory, vol. 42, issue 2 , 1996.

[13] J.B. De Long and A. Shleifer and L.H. Summers and R.J. Waldmann, "Noise Trader Risk in Financial Markets", Journal of Political Economy, vol. 98, issue 4, 1990, pp 703-738.

[14] S. Iwarere and B.R. Barmish, "A confidence interval triggering method for stock trading via feedback control", American Control Conference, 2010.

[15] S. Malekpour and B.R. Barmish, "A Drawdown Formula for Stock Trading Via Linear Feedback in a Market Governed by Brownian Motion”, European Control Conference, 2013.

[16] S. Malekpour and B.R. Barmish, "How Useful are Mean-Variance Considerations in Stock Trading via Feedback Control?", Proceedings of the IEEE Conference on Decision and Control, 2012.

[17] L. Mekhoff, "The noise trading approach: Questionnaire evidence from foreign exchange", Journal of International Money and Finance, vol. 17, issue 3, 1998, pp 547-564.

[18] S. Mudchanatongsuk and J.A. Primbs and W. Wong, "Optimal Pairs Trading: A Stochastic Control Approach", American Control Conference, 2008.

[19] J.A. Primbs and B.R. Barmish, "An Introduction to Hedged-Like Stock Trading from a Control Theoretic Point of View", American Control Conference Tutorial Session, 2012.

[20] J.A. Primbs and C.H. Sung, "Stochastic Receding Horizon Control of Constrained Linear Systems With State and Control Multiplicative Noise", IEEE Transactions on Automatic Control, vol. 54, issue 2, 2009

[21] R.J. Shiller, "Do stock prices move to much to be justified by subsequent changes in dividends?", The American Economic Review, vol. 71, issue 3, 1981, pp 421-436.

[22] M.P. Taylor and H. Allen, "The use of technical analysis in the foreign exchange market", Journal of International Money and Finance, vol. 11, issue 3, 1992, pp 304-314. 